\title{
HF-induced airglow at magnetic zenith: theoretical considerations
}

\author{
E. V. Mishin ${ }^{1}$, W. J. Burke ${ }^{2}$, and T. Pedersen ${ }^{2}$ \\ ${ }^{1}$ Boston College, Institute for Scientific Research, Chestnut Hill, Massachusetts, USA \\ ${ }^{2}$ Air Force Research Laboratory, Space Vehicles Directorate, Hanscom Air Force Base, MA 01731, USA
}

Received: 18 September 2003 - Revised: 19 December 2003 - Accepted: 14 January 2004 - Published: 31 January 2005

Part of Special Issue "Eleventh International EISCAT Workshop"

\begin{abstract}
Observations of airglow at $630 \mathrm{~nm}$ (red line) and $557.7 \mathrm{~nm}$ (green line) during HF modification experiments at the High Frequency Active Auroral Research Program (HAARP) heating facility are analyzed. We propose a theoretical framework for understanding the generation of Langmuir and ion acoustic waves during magnetic zenith injections. We show that observations of HF-induced airglow in an underdense ionosphere as well as a decrease in the height of the emitting volume are consistent with this scenario.
\end{abstract}

Key words. Space plasma physics (active perturbation experiments; nonlinear phenomena; wave-wave interactions)

\section{Introduction}

It is well known that injections of high-power, highfrequency (HF) radio waves strongly perturb ionospheric plasmas. The excitation of airglow at 630.0 and $557.7 \mathrm{~nm}$ is a distinctive, visual feature of such HF-induced perturbations (e.g. Sipler et al., 1974; Bernhardt et al., 1989; Pedersen and Carlson, 2001; Gustavsson et al., 2001, 2002; Kosch et al., 2000, 2002a,b; Pedersen et al., 2003). The red $(r)$ and green $(g)$ lines are emitted by atomic oxygen in the $O\left({ }^{1} D\right)$ and $O\left({ }^{1} S\right)$ states with the excitation energies $\varepsilon_{r}=1.96 \mathrm{eV}$ and $\varepsilon_{g}=4.17 \mathrm{eV}$, respectively.

Significantly, green-to-red ratios $\varsigma_{g r}>0.3$ are often observed. Gustavsson et al. (2002) emphasized that $\zeta_{g r}>0.1$ requires unrealizable electron temperatures $T_{e}>2 \mathrm{eV}$, pointing out the importance of electron acceleration in the highenergy tail. The latter stems from resonant interactions with plasma turbulence generated by heater waves. Stochastic interactions heat the bulk of electrons (e.g. Gurevich et al., 1985; Dimant et al., 1992; Mantas and Carlson, 1996; Gurevich and Milikh, 1997; Istomin and Leyser, 2003). Both effects are well documented (e.g. Carlson et al., 1982; Gustavsson et al., 2001; Rietveld et al., 2003).

Observations from the heating facilities at Troms $\varnothing$ $\left(\simeq 69.7^{\circ} \mathrm{N}, \simeq 18.9^{\circ} \mathrm{E}\right)$ and Gakona, Alaska $\left(\simeq 62.4^{\circ} \mathrm{N}\right.$,

Correspondence to: E. V. Mishin

(evgenii.mishin@hanscom.af.mil) $145.15^{\circ} \mathrm{W}$ ) show that the HF-induced airglow maximizes during injections toward magnetic zenith (MZ) (Kosch et al., 2002a; Pedersen et al., 2003). The same is true for the intensity of Langmuir waves (Isham et al., 1999) and electron heating (Rietveld et al., 2003; Dhillon and Robinson, 2003 ) observed by the EISCAT UHF ( $f_{0}=933 \mathrm{MHz}$ ) radar. Furthermore, the red line at MZ is excited at extremely low effective radiative power (ERP) $P_{0} \sim 2 \mathrm{MW}$ (Pedersen et al., 2003). The power of ion acoustic waves observed by the EISCAT UHF radar was found to be enhanced at, and $6^{\circ}$ to the south of, MZ (Dhillon and Robinson, 2003).

The generation of Langmuir $(l)$ and ion acoustic waves in HF modification experiments is usually explained in terms of the parametric decay (PDI) or oscillating two-stream (OTSI) instabilities of ordinary $(o)$ mode heating waves (Fejer, 1979). However, $o$-mode waves with incident angles $\theta_{0}$ outside the Spitze region, $\theta_{0}>\theta_{c}$, reflect at altitude $H_{m z}$ a few $\mathrm{km}$ below the "standard" reflection altitude $H_{0}$, where the local plasma frequency $f_{p}\left(H_{0}\right) \simeq 10^{4} \sqrt{n_{e}\left(H_{0}\right)}$ Hz equals the injected frequency $f_{0}$. Here, $\theta_{c}=\arcsin \left(\sqrt{\frac{f_{c}}{f_{c}+f_{0}}} \sin \chi\right), f_{c}$ is the electron gyrofrequency at an altitude of $200 \mathrm{~km}, n_{e}$ is the electron density in $\mathrm{cm}^{-3}$, and $\chi$ is the conjugate of the magnetic dip angle. Figure 1 shows a schematic of regions near $o$-mode reflection at geographic (GZ) and magnetic zenith at Gakona for $f_{0}=7.8 \mathrm{MHz}$.

For a heater ERP $P_{0}=150 \mathrm{MW}$ and distance $R=250 \mathrm{~km}$, the wave amplitude is $E_{0} \simeq 5.5 \sqrt{P_{0}} / R \simeq 0.27 \mathrm{~V} / \mathrm{m}$ (swelling is absent). With $\widetilde{f_{0}}=f_{0}[\mathrm{MHz}]=6$ and $T_{e}=0.1 \mathrm{eV}$, the HF energy density at the reflection point is $W_{0}=\frac{E_{0}^{2}}{4 \pi} \sim 10^{-4} n_{e} T_{e}$. It is sufficient to drive the PDI at $H_{0}$ (Fejer, 1979) but is not enough for injections toward MZ due to a mismatch of frequencies at $H_{m z}$ and beneath.

Gurevich et al. (2002) suggested that decreased plasma densities, within striations generated by heating waves, permit the phase matching necessary for the PDI/OTSI to develop. Istomin and Leyser (2003) showed that electrons can be accelerated by upper hybrid waves trapped inside striations. Changes in the refraction index (self-focusing), caused by striations that form within tens of seconds after turnon, explain some features of the spatial distribution of the 


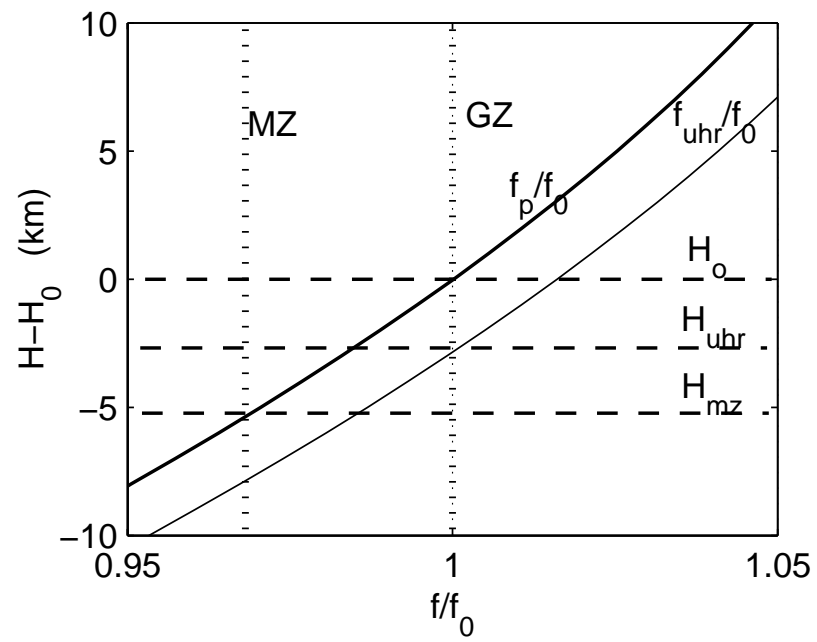

Fig. 1. Schematic of regions near ordinary-mode reflection for vertical and magnetic field-aligned heater pointings. Solid lines designate the plasma (heavy line) and upper hybrid resonance (thin) frequencies; vertical dotted lines show the cutoff frequencies at GZ and $\mathrm{MZ}$, respectively; horizontal dashed lines indicate the heights of reflection at GZ, the upper hybrid resonance, and MZ.

HF-induced airglow. Kosch et al. (2002b) showed that the intensity of red-line emissions decreased when the heating frequency approached an electron gyro-harmonic, pointing out the importance of upper hybrid turbulence at MZ.

On the other hand, Langmuir waves at MZ were seen within $\sim 10 \mathrm{~ms}$ (Isham et al., 1999). In 5-s resolution observations made by Dhillon and Robinson (2003), the initial increase in UHF backscatter, characterized by an overshoot, was seen coincident with the heater turn-on. Electron temperatures generally increased at heater turn-on, but the maximum increase occurred several seconds later. Mishin et al. (2004) show that during injections toward magnetic zenith the intensities of the green-line emission gained $\sim 5 \mathrm{R}$ (Rayleighs) within $\sim 1 \mathrm{~s}$. This period is dubbed the onset of HF-induced airglow. Time scales $\lesssim 1 \mathrm{~s}$ appear too short for significant striations to develop (e.g. Gondarenko et al., 1999).

Striations are generated near the upper hybrid resonance height $H_{\mathrm{uhr}}$, where $f_{0}=f_{\mathrm{uhr}}=\sqrt{f_{p}^{2}+f_{c}^{2}}$ (e.g. Vaskov et al., 1981; Lee and Kuo, 1983). Hence, the reflection height of an obliquely-injected heating wave must be at least at the same level. For reflection to occur above $H_{\mathrm{uhr}}$ during MZ injections, the inequality $f_{c}>f_{0} \cdot \sin \chi$ must be met. For MZ injections at the HAARP heating facility $\left(\chi \simeq 14.6^{\circ}\right), H_{\mathrm{uhr}}<H_{m z}$ if $f_{0}<f_{0} \chi \simeq 5.4 \mathrm{MHz}$. However, the strongest airglow occurred at higher $f_{0}$ values (Pedersen et al., 2003) and at altitudes well below $H_{0}$ and $H_{\text {uhr }}$ (Kosch et al., 2002a).

Mishin et al. (2004) suggested a scenario for exciting plasma turbulence with subsequent electron heating and acceleration in the HF-illuminated region at MZ. This scenario, depicted in Fig. 2, explores the results of Kuo et al. (1997), who showed that the OTSI can be excited by upper hybrid

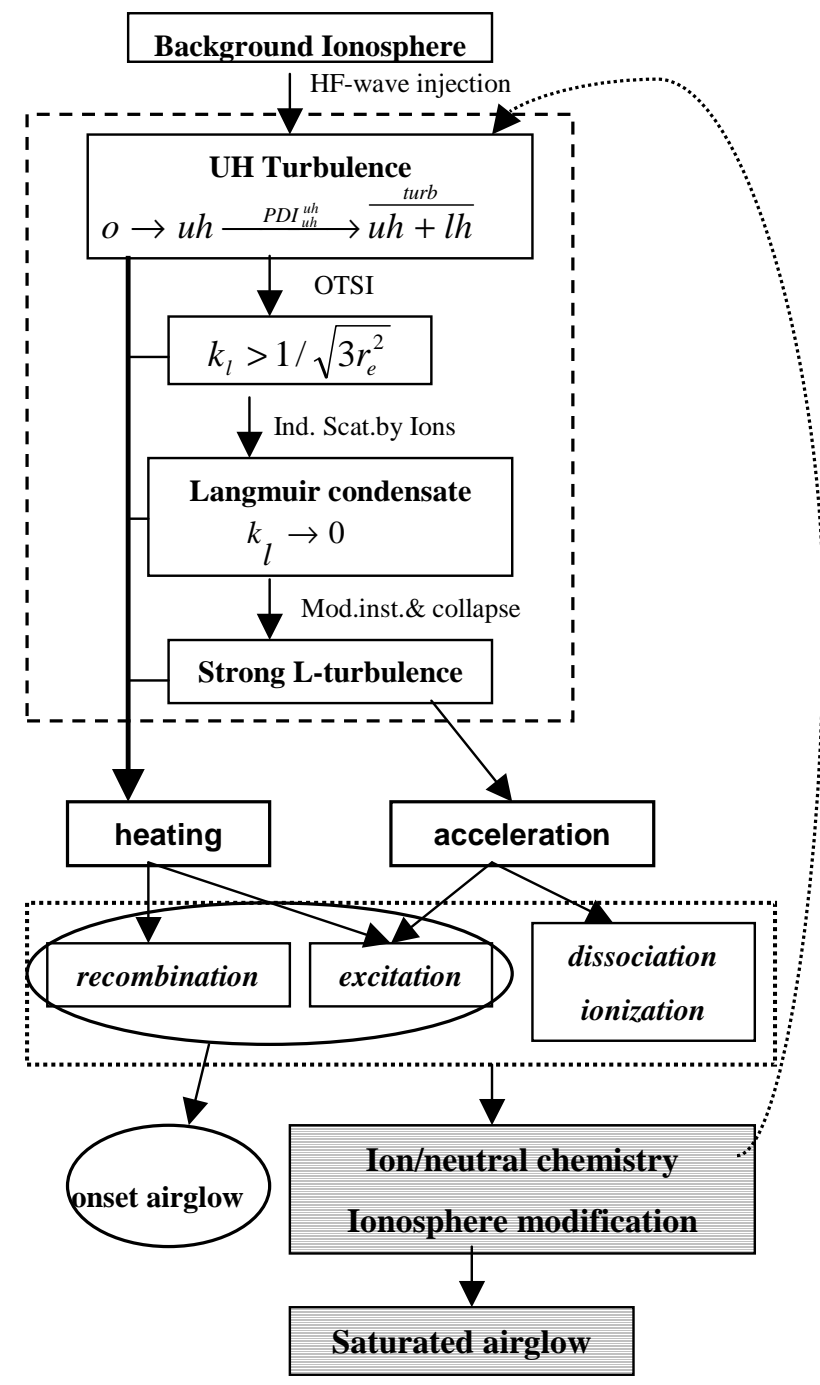

Fig. 2. Block-diagram from Mishin et al. (2004) showing energy flow in a model of HF-induced airglow at MZ. Shaded boxes indicate the steps to be worked out.

( $u h$ ) waves. In turn, $u h$-waves are generated through the linear conversion of the $o$-mode on pre-existing field-aligned irregularities (Wong et al., 1981) or by the parametric decay $o \rightarrow u h+l h\left(\mathrm{PDI}_{o}^{u h}\right)($ e.g. Istomin and Leyser, 1995); $l h$ stands for lower hybrid waves.

The conversion process occurs at $H_{\mathrm{uhr}}$ and has no threshold. $\mathrm{PDI}_{o}^{u h}$ develops if $E_{0}>E_{0}{ }^{u h} \simeq 1.6 \widetilde{f}_{0}^{2} \mathrm{mV} / \mathrm{m}$, provided the corresponding matching conditions are met for $x_{u h}=k_{u h}^{2} r_{e}^{2}<1$. Here, $r_{e}$ is the thermal electron gyroradius and $k_{u h}$ is the $u h$-wave vector. Otherwise, Landau damping of short-scale lower hybrid waves raises the threshold value (Mishin et al., 1997). The rise time of the (primary) $u h$-wave is $\tau_{u h} \sim 1-3 \mathrm{~ms}$. When the amplitude of the primary $u h$-wave $E_{u h}$ exceeds $\sim 10 \mathrm{mV} / \mathrm{m}$, it parametrically decays into lower frequency $u h$-wave (Zhou et al., 1994). The same is true for subsequently-generated $u h$-waves. 
The OTSI excited by $u h$-waves produces short-scale, $k_{l}>3^{-1 / 2} / r_{e}$, Langmuir waves (Kuo et al., 1997). These waves saturate via spectral transfer toward small $k_{l}$, due to induced scattering by ions (e.g. Zakharov et al., 1976). Depending on the energy density of the $u h$-turbulence, which depends on the ERP, the induced scattering process may deliver the Langmuir wave energy $W_{l}$ to the region $k_{l} \rightarrow 0$ (Langmuir condensate) (Zakharov et al., 1976). The dynamics of the Langmuir condensate is defined by the modulational instability and collapse, leading to the establishment of strong (cavitating) Langmuir turbulence (e.g. Zakharov, 1972). This makes the generation of Langmuir turbulence possible within $\sim 10 \mathrm{~ms}$, consistent with the Isham et al. (1999) observations.

Collisional damping of high-frequency plasma waves is the major source of electron heating. Resonant $l \mathrm{~h}$ - and $l$ wave-particle interactions accelerate electrons. Based on this scenario, Mishin et al. (2004) developed an explanatory model for the growth of emissions from MZ within the first few seconds after the HF transmitter turn on (airglow onset). The model accounts for the roles played by ambient photoelectrons and dissociative recombination of oxygen ions and shows that heating and acceleration of ambient electrons can explain the observed features of the airglow onset at MZ.

A consistent theory of the HF-induced airglow, accounting for the ion/neutral chemistry, modification of the heated spot, and self-focusing, has yet to be worked out. Pedersen et al. (2003) mentioned that the brightest emissions had a slight tendency to occur very near the critical frequency of the Flayer $f_{o} \mathrm{~F} 2$, but all emissions cut off sharply at about $0.5 \mathrm{MHz}$ above foF2.

This paper continues our analysis of optical data acquired during the February 2002 campaign at the HAARP heating facility. We focus on intervals when foF 2 dropped below the heating frequency, but HF-induced green and red lines at MZ remained. We characterize such events as the HF-induced airglow in an underdense ionosphere and show that this feature can be understood in terms of the above scenario.

\section{Airglow at HAARP in the underdense ionosphere}

Figure 3 shows all-sky images of the HF-induced red- and green-line emissions observed from the HAARP site during a long, $30 \mathrm{~min}$, pulse that began at 04:32:12 UT on 13 February 2002 (Pedersen et al., 2003). The data show one of the most intense green-line emissions observed at HAARP. During this (twilight) period $o$-mode waves were injected toward magnetic zenith at $f_{0}=7.8 \mathrm{MHz}$ and at full power of $940 \mathrm{KW}$ (ERP $P_{0} \simeq 165 \mathrm{MW}$ ). Exposure durations were $7.5 \mathrm{~s}$.

Figure 4 (top panel) shows the variation of the red- and green-line intensities in the course of the long pulse. The data points are from two red line exposures made at 0 and $24 \mathrm{~s}$ after each minute, with one $557.7 \mathrm{~nm}$ exposure at $12 \mathrm{~s}$. Simultaneous observations from a digisonde located at the HAARP site showed that the reflection height of a $7.8-\mathrm{MHz}$ $o$-mode wave at MZ gradually increased from $H_{m z} \sim 235$ to $\sim 270 \mathrm{~km}$ between 04:30 and 04:50 UT. At the same time, the shadow height at the HAARP site increased from $\sim 230$ to $\sim 300 \mathrm{~km}$ following the increasing solar zenith angle and so does the height of the F-peak $h m \mathrm{~F} 2$. The magnetic conjugate point was sunlit, maintaining a flux of energetic photoelectrons (e.g. Doering et al., 1975; Peterson et al., 1977) above HAARP most of the time.

To account for natural (background) variations, a polynomial fit was made to intensities measured along the magnetic meridian in each all-sky image, with the heated area blocked out. The heater-induced airglow is determined as the difference $\Delta_{\lambda}=I_{\lambda}-b_{\lambda}$ between the airglow from the heated volume $I_{\lambda}$ and the background airglow $b_{\lambda}$. During heater-off periods, $\Delta_{\lambda}^{(o f f)}$ was less than $\sim 3-5$ and $\sim 7-10 \mathrm{R}$ for greenand red-line emissions, respectively. This small difference determines the accuracy of heater-induced airglow measurements.

The critical frequency of the F-layer is determined from 5min digisonde observations at the HAARP site. Apparently, foF 2 dropped below the transmitter frequency in the course of this pulse. However, the airglow did not diminish. Figure 5 shows that the HF-induced airglow disappeared only at $f o \mathrm{~F} 2<f o \mathrm{~F} 2^{c} \simeq f_{0}-0.5 \mathrm{MHz}$. A remark is in order. One can argue that the HAARP digisonde points vertically and the actual foF 2 at $H_{m z}$, that is about $50 \mathrm{~km}$ to the south of the local vertical, might be significantly different. However, this is unlikely, for the local geophysical conditions were very quiet. This conjecture is supported by the small difference between the airglow from the heated volume during heater-off periods and the (barely changing) background airglow about $50 \mathrm{~km}$ apart.

In addition to Figs. 3 and 5, Fig. 6 shows the results of a survey of all the airglow-events with a 50-R or more enhancement in red line emissions observed on clear-sky nights during the February 2002 optics campaign. Apparently, for $f_{0} \geq 5.8 \mathrm{MHz}$ airglow is enhanced by heating waves until $f o \mathrm{~F} 2 \geq f o \mathrm{~F} 2 c$. No enhancements were observed at $f o \mathrm{~F} 2<f o \mathrm{~F} 2{ }^{c}$. At $f_{0}=4.8$ and $4.5 \mathrm{MHz}$ the cutoff frequency coincides with $f_{0}$ accurate to within $\pm 0.1 \mathrm{MHz}$, that is within the accuracy of determination of $f_{o} \mathrm{~F} 2$. It is worth mentioning that the difference between $f_{p}\left(H_{m z}\left(f_{0}\right)\right)$ and $f_{0}=7.8$, $6.8,5.8$, and $4.8 \mathrm{MHz}$ is about $-0.24,-0.2,-0.17$, and $-0.14 \mathrm{MHz}$, respectively.

\section{Discussion}

Mishin et al. (2004) emphasized that the scenario in Fig. 2 works at and below the reflection layer $H_{m z}$, provided that the matching conditions for $\mathrm{PDI}_{o}^{u h}$ (e.g. Istomin and Leyser, 1995) are satisfied at local values of $x_{u h}=k_{u h}^{2} r_{e}^{2}<1$. During injections toward $\mathrm{MZ}$ standing waves do not form, and the heating wave amplitude at the reflection point does not increase relative to that at lower altitudes. Thus, the reflection layer does not stand out as it does for injections within the Spitze region, where the Airy pattern is formed. 


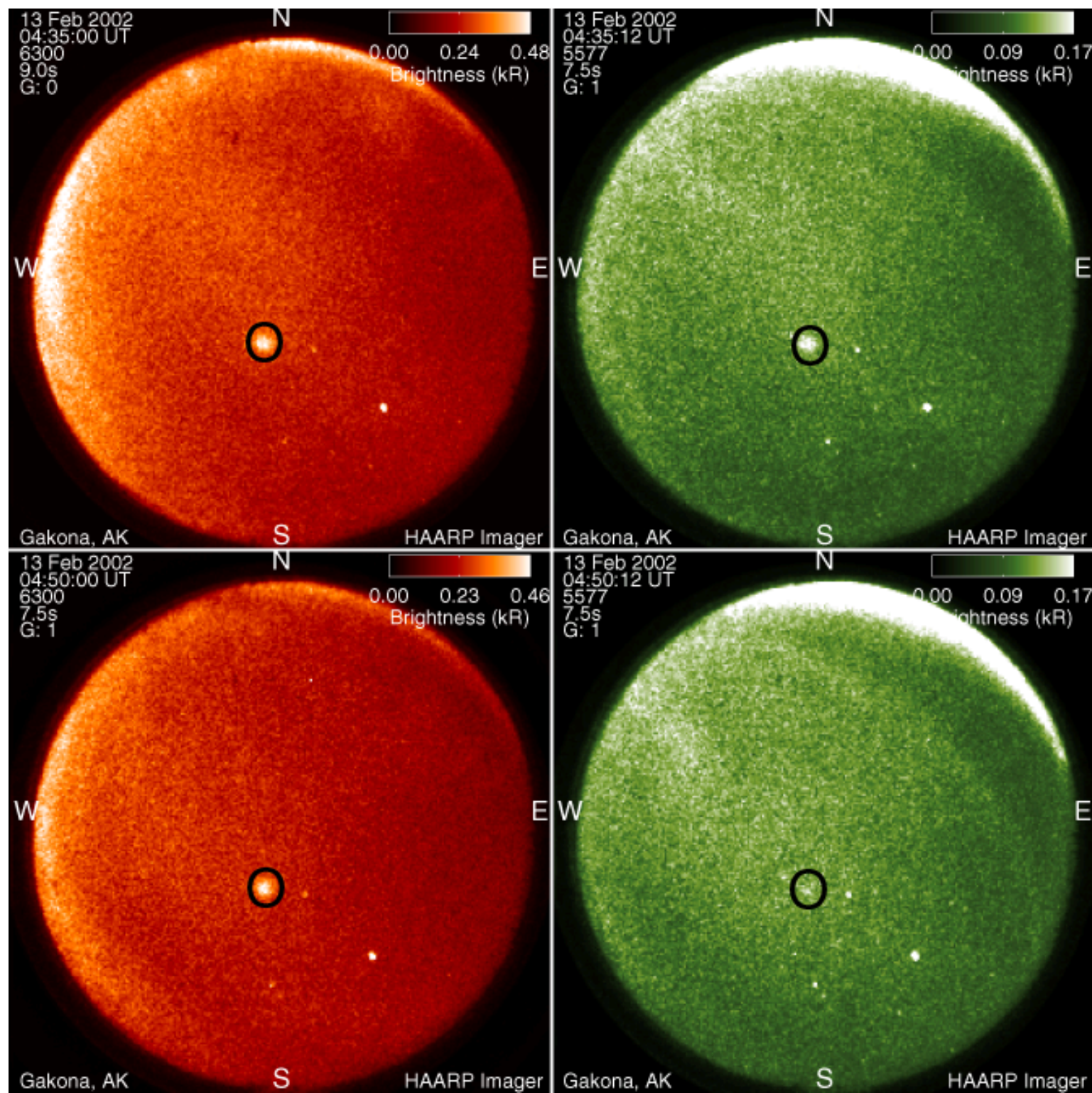

Fig. 3. Heater-induced red- and green-line emissions during the long pulse before (top) and after (bottom) foF 2 has dropped below $f_{0}$. Bold circles mark the heated spot at MZ. Bright dots outside the circles are stars. The color bars show the brightness from 0 to $0.48 \mathrm{kR}$ and from 0 to $0.17 \mathrm{kR}$ for the red- and green-line emissions, respectively.

The ERP and angular width of the HF beam from the HAARP heater depend on the radiated frequency. Roughly, one can estimate $P_{0} \propto f_{0}^{2}$, while the beam has full width at half maximum $\sim 27^{\circ}$ and $\sim 15^{\circ} \mathrm{N}-\mathrm{S}$ at $3.3 \mathrm{MHz}$ and at $7.8 \mathrm{MHz}$, respectively. For $f_{0}=7.8,6.8,5.8,4.8$, and $4.5 \mathrm{MHz}$, the free space field of the incident wave from the HAARP heater is $E_{0} \simeq 0.25,0.2,0.18,0.15$, and $0.14 \mathrm{~V} / \mathrm{m}$, that is $E_{0}>E_{0}^{u h}\left(f_{0}\right)$.

From the dispersion relation of $u h$ waves

$$
\begin{aligned}
f_{u h}\left(x_{u h}\right) \simeq & f_{\mathrm{uhr}}+\frac{3}{2} f_{c} \frac{\left(s^{2}-1\right)}{s\left(s^{2}-4\right)} x_{u h}+ \\
& \frac{\left(s^{2}-1\right)^{2}}{2^{s+1} s !} \frac{f_{c}^{2}}{f_{u h}\left(x_{u h}\right)-s f_{c}} x_{u h}^{s-1}
\end{aligned}
$$

one can readily obtain the value of the $u h$-wave vector necessary for $\mathrm{PDI}_{o}^{u h}$ to occur at a given altitude $H$ (cf. Istomin and Leyser, 1995)

$$
x_{u h}^{*}\left(H, f_{0}\right) \simeq \frac{2}{3} \frac{s\left(s^{2}-4\right)}{s^{2}-1} \frac{f_{0}-f_{\mathrm{uhr}}(H)}{f_{c}(H)} .
$$

This assumes that $f_{0}$ is no closer than $\sim 0.006 f_{c}$ to an electron gyro-harmonic $s \cdot f_{c}$ and $x_{u h}<0.5$. Here, $s$ is defined by rounding up the $f_{\mathrm{uhr}} / f_{c}$ ratio to the nearest integer. Given $f_{c}\left(H_{m z}\right) \simeq 1.37 \mathrm{MHz}$ at the HAARP site (from the IGRF geomagnetic field model), the heating frequencies amount to $\simeq f_{c} \cdot 5.7,4.96,4.23,3.5$, and 3.3 , respectively. Apparently, $f_{0}-s f_{c}>0.006 f_{c}$. At $H_{m z}$, corresponding $s$-values are 6(-), 


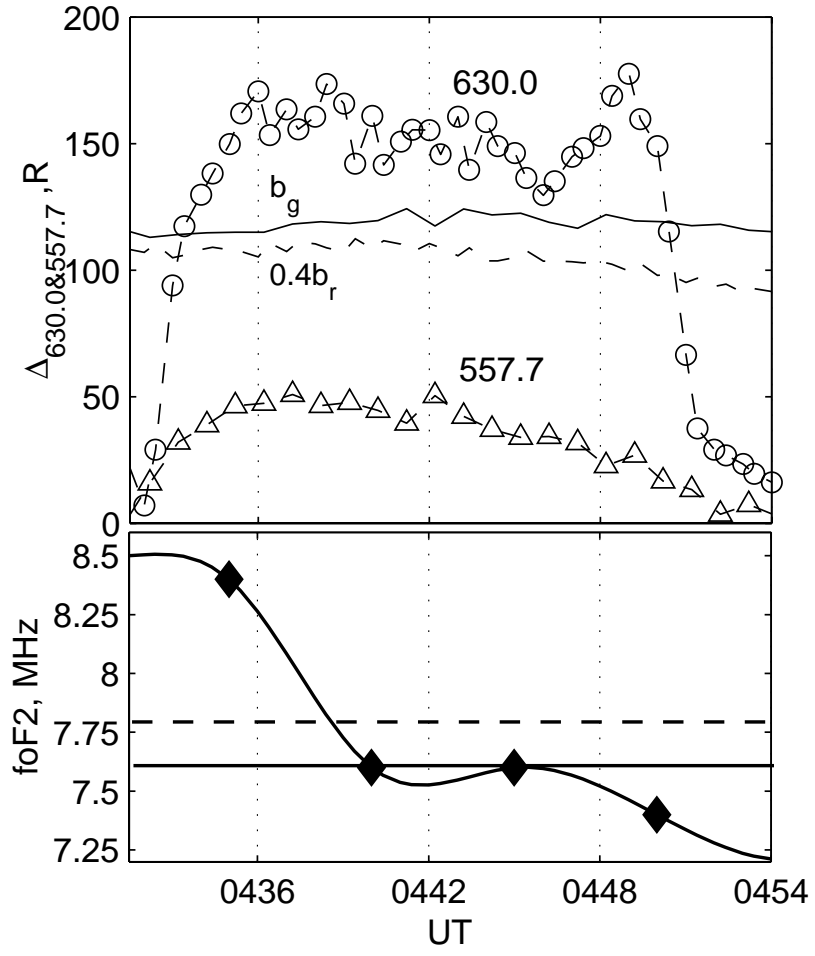

Fig. 4. Heater-induced red- and green-line emissions during the long pulse on 13 February 2002. Solid and dashed lines show intensities of the background green $\left(b_{g}\right)$ and (scaled) red $\left(b_{r}\right)$ emissions, respectively. (bottom) The critical frequency of the F-layer above the HAARP site (diamonds); the solid line represents a spline approximation; dashed and dotted lines show the heating frequency and the plasma frequency at $H_{m z}$, respectively.

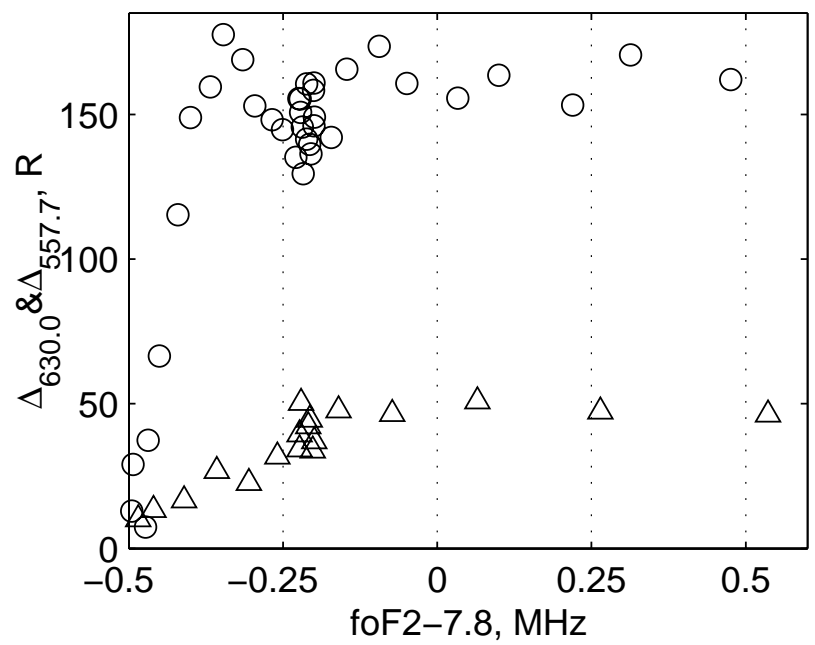

Fig. 5. Variation of the HF-induced red- (circles) and green-line (triangles) intensities with foF 2 during the long pulse shown in Fig. 4.

$5(-), 4(+), 4(-)$, and $3(+)$, respectively; $( \pm)$ stands for the sign of $f_{u h}-s f_{c}$.

Let us first consider the case $f o \mathrm{~F} 2>f_{0}>f_{0}^{\chi} \simeq 5.5 \mathrm{MHz}$, that is $H_{m z}\left(f_{0}\right)<H_{\text {uhr }}\left(f_{0}\right)$. As follows from Eq. (2), the

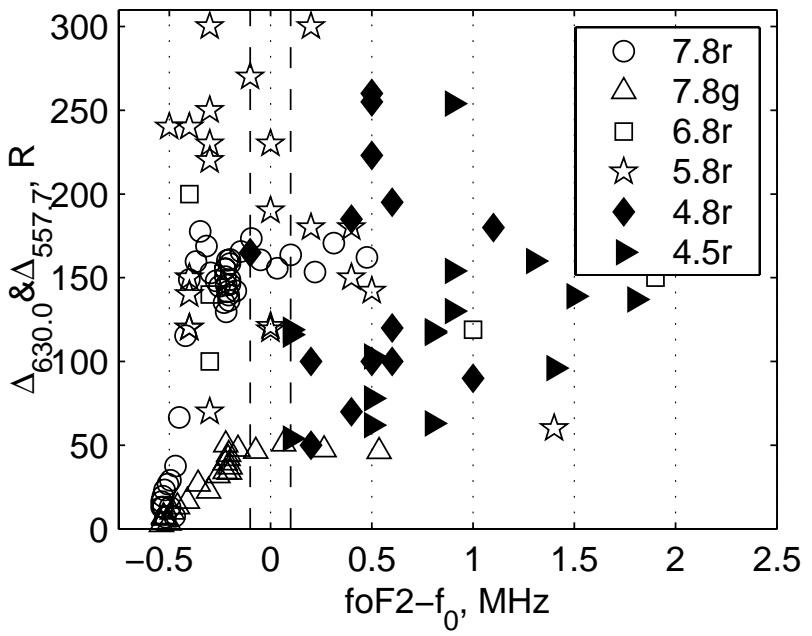

Fig. 6. Summary plot of the variation of the HF-induced airglow at $\mathrm{MZ}$ with $f o \mathrm{~F} 2-f_{0}$. Here $7.8, \ldots 4.5$ stand for $f_{0}=7.8, \ldots 4.5 \mathrm{MHz}$; $r$ and $g$ indicate red- and green-line emissions; vertical dashed lines show $\pm 0.1 \mathrm{MHz}$ interval.

matching conditions for $f_{0}=7.8,6.8$, and $5.8 \mathrm{MHz}$ at the reflection height $H_{m z}\left(f_{0}\right)$ are satisfied at $x_{u h}^{*}\left(f_{0}\right)<0.5$. Importantly, Eq. (2) holds well below $H_{m z}\left(f_{0}\right)$, that is $x_{u h}^{*} \rightarrow 1$ at $H^{*}\left(f_{0}\right)=H_{m z}\left(f_{0}\right)-\delta H_{m z}\left(f_{0}\right)$. Here, $\delta H_{m z}\left(f_{0}\right)$ amounts to $\simeq 3.5,6$, and $11.4 \mathrm{~km}$, respectively (the gradient scale-length of a linear plasma density profile of $50 \mathrm{~km}$ is assumed).

The volume emission rate (VER) of the red-line emission $\eta_{r}$ is mainly defined by the electron temperature altitudeprofile (e.g. Mantas and Carlson, 1996; Mishin et al., 2004). It is very sensitive to the distribution of thermal electrons (TEDF), which may strongly deviate from a Maxwellian distribution (MD) due to inelastic collisions with molecular nitrogen $N_{2}$ (Mishin et al., 2000). For $n_{e}$ given, the TEDFMD-deviation decreases with altitude as well as collisional deactivation of the $O\left({ }^{1} D\right)$ state. Both effects provide increasing $\eta_{r}\left(T_{e}\right)$ in the course of the twilight observations (Mishin et al., 2000, 2004). Thus, following $H_{m z}$, the redline intensity is expected to rise when $H_{m z}$ approaches $h m \mathrm{~F} 2$ or $f o \mathrm{~F} 2 \rightarrow f_{p}\left(H_{m z}\right)$.

On the other hand, the VER of the green-line emission $\eta_{g}$ is dominated by suprathermal electrons and increases at lower altitudes (Mishin et al., 2004). Therefore, the greenline intensity is expected to decrease when $f o \mathrm{~F} 2$ approaches $f_{p}\left(H_{m z}\right)$. These conjectures are consistent with data in Figs. 5 and 6.

As heating frequencies diminish with time, following $f o \mathrm{~F} 2$, the heated volume rises following $\delta H_{m z}\left(f_{0}\right)$. Thus, for the same ERP, $P_{0}$, one should expect $\Delta_{630.0}\left(f_{0}\right)$ to increase with decreasing $f_{0}>f_{0}^{\chi}$ in an underdense ionosphere. Indeed, from Fig. 6 follows that $\Delta_{630.0}(5.8)>\Delta_{630.0}(6.8)$ and $\Delta_{630.0}(7.8)$ even despite the dependence $P_{0} \propto f_{0}^{2}$.

After $f o \mathrm{~F} 2$ drops below $f_{p}\left(H_{m z}\right)$, heating waves injected toward MZ are not reflected. The only consequence is that diminishing $f_{\mathrm{uhr}}(H)$ causes $x_{u h}^{*}(H)$ (Eq. (2)) to increase. 
$\mathrm{PDI}_{o}^{u h}$ develops if the dispersion curve (Eq. (1)) intersects with $f_{0}$ at $x_{u h}(H)<1$. This is possible for $f_{0} \simeq 5.8 \mathrm{MHz}$ $\left(4.23 f_{c}\right)$ only if $f_{\text {uhr }}>4.006 f_{c}$ or $f_{p}>5.31 \mathrm{MHz}$. Apparently, the cutoff frequency is $\approx f o \mathrm{~F}^{c}$. At first glance, the cutoff frequencies for $f_{0}=7.8$ and $6.8 \mathrm{MHz}$ can be roughly defined by the condition $x_{u h}^{*}\left(h m \mathrm{~F} 2, f_{0}\right) \sim 1$. More precisely, one has to account for the second term in Eq. (1) that limits $f_{u h}\left(x_{u h}\right)-f_{\text {uhr }}$ and determines the location of its maximum $\sim f_{c}^{2} / 2 f_{\text {uhr }}$ at $x_{u h}<1$ (e.g. Istomin and Leyser, 1995, 2003). As a result, the cutoff frequencies turn out to be close to $f o \mathrm{~F} 2^{c} \simeq f_{0}-0.5 \mathrm{MHz}$.

For $f_{0}<f_{0}^{\chi}$, that is 4.8 and $4.5 \mathrm{MHz}, H_{m z}$ is located above the upper hybrid resonance layer and Eq. (2) can be satisfied at $H_{\text {uhr }}$ and by $\sim 15 \mathrm{~km}$ below. In this case we expect striations to grow from $H_{\mathrm{uhr}}$. This process is expected to be most efficient near the maximum of the F-peak $\left(f_{\mathrm{uhr}}=f_{0} \rightarrow f o \mathrm{~F} 2\right)$, where the vertical plasma density gradient is minimum. Developed striations disguise the plasma density profile thereby making it difficult for $\mathrm{PDI}_{o}^{u h}$ and OTSI to develop below $H_{\text {uhr }}$. As the generation of plasma turbulence is prohibited at lower altitudes, the heater-induced airglow is dominated by heating inside striations (Gurevich et al., 2002) and hence must vanish whenever $f o \mathrm{~F} 2$ drops below $f_{0}$.

\section{Conclusion}

The results of an ongoing analysis of optical data acquired during the February 2002 campaign at the HAARP heating facility are presented. Here we focus on observations of the underdense ionosphere, when $f o \mathrm{~F} 2$ drops below the heating frequency but HF-induced green and red lines at MZ remained. We show that this feature can be understood in terms of the scenario for the generation of strong turbulence at magnetic zenith with the OTSI of upper hybrid waves as the primary source of Langmuir waves.

Acknowledgements. This research was supported in part by the HF Active Auroral Research Program (HAARP) under AFRL contract F19628-02-C-0012 with Boston College and by Air Force Office of Scientific Research under tasks 2311AS and 2311SD.

Topical Editor M. Lester thanks R. Dhillon and another referee for their help in evaluating this paper.

\section{References}

Bernhardt, P., Tepley, C., and Duncan, L.: Airglow enhancements associated with plasma cavities formed during ionospheric heating experiments, J. Geophys. Res., 94, 9071-9092, 1989.

Carlson, H., Wickwar, V., and Mantas, G.: Observations of fluxes of suprathermal electrons accelerated by HF excited Langmuir instabilities, J. Atm. Terr. Phys., 12, 1089-1100, 1982.

Dhillon, R. and Robinson, T.: Observations of time dependence and aspect sensitivity of regions of enhanced UHF backscatter associated with heating, in Abstracts from the $11^{\text {th }}$ Biannual EISCAT Workshop , 116, SRI International, Menlo Park, CA, USA, August 2003 .
Dimant, Y., Gurevich, A., and Zybin, K.: Acceleration of electrons under the action of intense radio-waves near electron cyclotron harmonics, J. Atm. Terr. Phys., 54, 425-436, 1992.

Doering, J., Peterson, W., Bostrom, C., and Armstrong, J.: Measurement of low-energy electrons in the day airglow and day side auroral zone from A tmosphere Explorer C, J. Geophys. Res., 80, 3934-3944, 1975.

Fejer, J.: Ionospheric modification and parametric instabilities, Rev. Geophys. Space Phys., 17, 135-153, 1979.

Gondarenko, N. A., Guzdar, P. N., Milikh, G. M., Sharma, A. S. Papadopoulos, K., and Ossakow, S. L.: Spatio-temporal development of filaments due to the thermal self-focusing instability near the critical surface in ionospheric plasmas, Izv. VUZ. Radiophys., 7, 589, 1999.

Gurevich, A. and Milikh, G.: Artificial airglow due to modifications of the ionosphere by powerful radio waves, J. Geophys. Res., 102, 1389-394, 1997

Gurevich, A., Dimant, Y., Milikh, G., and Vaskov, V.: Multiple acceleration of electrons in the regions high-power radio-wave reflection in the ionosphere, J. Atmos. Terr. Phys., 47, 1057-1070, 1985.

Gurevich, A., Zybin, K., Carlson, H., and Pedersen, T.: Magnetic zenith effect in ionospheric modifications, Phys. Lett. A, 305, 5 , 264-274, 2002.

Gustavsson, B., Brändström, B., Steen, A., Sergienko, T., Leyser, T., Rietveld, M., Aso, T., and Erjii, M.: Nearly simultaneous images of HF-pump enhanced airglow at $6300 \AA$ and $5577 \AA$, Geophys. Res. Lett., 29, 2220, doi: 10.1029/2002GL015350, 2002.

Gustavsson, B., Sergienko, T., Rietveld, M. T. et al.: First tomographic estimate of volume distribution of HF-pump enhanced airglow emission, J. Geophys. Res., 106, 29 105-29 124, 2001.

Isham, B., Rietveld, M., Hagfors, T., LaHoz, C., Mishin, E., Kofman, W., Leyser, T., and van Eyken, A.: Aspect angle dependence of HF enhanced incoherent backscatter, Adv. Space Res. 24, 8, 1003-1006, 1999.

Istomin, Y. and Leyser, T.: Electron acceleration by cylindrical upper hybrid oscillations trapped in density irregularities in the ionosphere, Phys. Plasmas, 10, 2962-2970, doi: 10.1063/1.1578637, 2003.

Istomin, Y. and Leyser, T.: Parametric decay of an electromagnetic wave near electron cyclotron harmonics, Phys. Plasmas, 2, 2084 2097, 1995.

Kosch, M., Rietveld, M., Hagfors, T., and Leyser, T.: High-latitude $\mathrm{HF}$-induced airglow displaced equatorward of the pump beam, Geophys. Res. Lett., 27, 2817-2820, 2000.

Kosch, M., Rietveld, M., Yeoman, T., Cierpka, K., and Hagfors, T.: The high-latitude artificial aurora of 21 February 1999: An analysis, Advances in Polar Upper Atmosphere Research, 16, 112, 2002a.

Kosch, M. J., Rietveld, M., Kavanagh, A. J., Davis, C., Yeoman, T., Honary, F., and Hagfors, T.: High-latitude pumpinduced optical emissions for frequencies close to the third electron gyro-harmonic, Geophys. Res. Lett., 29, 2112, doi: 10.1029/2002GL015,744, 2002b.

Kuo, S., Lee, M., and Kossey, P.: Excitation of oscillating two stream instability by upper hybrid pump in ionospheric heating experiments at Troms $\varnothing$, Geophys. Res. Lett., 24, 2969-2972, 1997.

Lee, M. and Kuo, S.: Excitation of upper hybrid waves by a thermal parametric instability, J. Plasma Phys., 30, 30 463, 1983.

Mantas, G. and Carlson, H.: Reinterpretation of the 6300- $\AA$ airglow enhancemments observed in the ionosphere heating experiments 
based on analysis of Plateville, Colorado, data, J. Geophys. Res., 101, 195-209, 1996.

Mishin, E., Hagfors, T., and Kofman, W.: On origin of outshifted plasma lines during HF modification experiments, J. Geophys. Res., 102, 27 265-27 269, 1997.

Mishin, E., Carlson, H., and Hagfors, T.: On the electron distribution function in the F-region and airglow enhancements during HF modification experiments, Geophys. Res. Lett., 27, 28572860, 2000.

Mishin, E., Hagfors, T., and Isham, B.: A generation mechanism of the topside incoherent backscatter during HF modification experiments at Troms $\emptyset$, Geophys. Res. Lett., 28, 479-482, 2001.

Mishin, E. V., Burke, W. J., and Pedersen, T.: On the onset of HF-induced airglow at magnetic zenith, J. Geophys. Res., 109, A02305, doi:10.1029/2003JA010205, 2004.

Pedersen, T. and Carlson, H.: First observations of HF heaterproduced airglow at the High Frequency Active Auroral Research Program facility: Thermal excitation and spatial structuring, Radio Sci., 36, 1013-1026, 2001.

Pedersen, T., McCarrick, M., Gerken, E., Selcher, C., Sentman, D., Carlson, H., and Gurevich, A.: Magnetic zenith enhancement of HF radio-induced airglow production at HAARP, Geophys. Res. Lett., 30, 4, 1169, doi: 1029/2002GL016096, 2003.

Peterson, W., Doering, J., Potemra, T., McEntire, R., and Bostrom, C.: Conjugate photoelectron fluxes observed on Atmosphere Explorer C, Geophys. Res. Lett., 4, 109-112, 1977.
Rietveld, M., Kosch, M., Blagoveshchenskaya, N., Kornienko, V., Leyser, T., and Yeoman, T.: Ionospheric electron heating, optical emissions and striations induced by powerful HF radio waves at high latitudes: Aspect angle dependence, J. Geophys. Res., 108, A4, 1141, doi:10.1029/2002JA009543, 2003.

Sipler, D., Enemark, E., and Biondi, M.: 6300-Å intensity variations produced by the Arecibo ionospheric modification experiment, J. Geophys. Res., 79, 4276-4280, 1974.

Vaskov, V. V., Gurevich, A., and Karashtin, A. N.: Thermal selffocusing instability of plasma waves near resonance, Geomagn. Aeronomy, English Transl., 21, 724-728, 1981.

Wong, A., Morales, G. J., Eggleston, D., Santoru, J., and Behnke, R.: Rapid conversion of electromagnetic waves to electrostatic waves in the ionosphere, Phys. Rev. Lett., 47, 1340-1343, 1981.

Zakharov, V.: Collapse of Langmuir waves, Sov. Phys. J E T P, Engl. Transl., 35, 908, 1972.

Zakharov, V., Musher, S., and Rubenchik, A.: Weak Langmuir turbulence of an isothermal plasma, Sov. Phys. J E T P., 42, 80-86, 1976.

Zhou, H., Huang, J., and Kuo, S.: Cascading of the upper hybrid/electron B ernstein wave in ionospheric heating experiments, Phys. Plasmas, 1, 3044-3052, 1994. 